ADP-94-2/M22

gr-qc/9407021

\title{
Global properties of static spherically symmetric charged dilaton spacetimes with a Liouville potential
}

\author{
S.J. PoletTi $^{\star}$ AND D.L. Wiltshire ${ }^{\dagger}$ \\ Department of Physics and Mathematical Physics, University of Adelaide, \\ Adelaide, S.A. 5005, Australia.
}

\begin{abstract}
We derive the global properties of static spherically symmetric solutions to the EinsteinMaxwell-dilaton system in the presence of an arbitrary exponential dilaton potential. We show that - with the exception of a pure cosmological constant 'potential' - no asymptotically flat, asymptotically de Sitter or asymptotically anti-de Sitter solutions exist in these models.
\end{abstract}

\footnotetext{
$\star$ E-mail: spoletti@physics.adelaide.edu.au

† E-mail: dlw@physics.adelaide.edu.au
} 


\section{Introduction}

There has been considerable interest recently in the properties of 'stringy' black holes: classical solutions of tree-level string effective actions, in which the Einstein action is supplemented by fields such as the axion, gauge fields, and the dilaton which couples in a non-trivial way to the other fields. In particular, dilaton black holes have been shown to have novel thermodynamic properties [1-3], and to behave like elementary particles in some scattering scenarios [4].

Unfortunately, much of the work on dilaton black holes to date has involved models with one serious deficiency: the dilaton has usually been assumed to be massless. It is widely believed, however, that the dilaton must aquire a mass through some symmetry breaking mechanism. Indeed, this is necessary in order to avoid long-range scalar forces which would

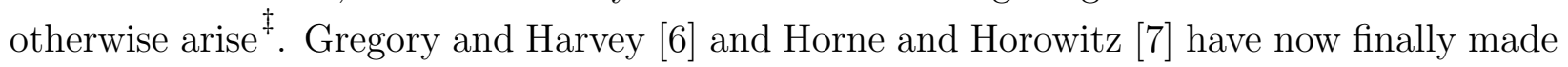
some investigation of black hole models which include a mass term - they have chosen a standard quadratic potential for the dilaton field ${ }^{\S}$. While a rigorous proof of the existence of black hole solutions in these models has still to be given, the arguments of Horne and Horowitz [7] are nonetheless compelling.

Ultimately, it would be physically desirable to investigate models of black holes in effective dilaton gravity theories in four dimensions which involve a dilaton potential generated by some specific symmetry breaking mechanism, rather than simply an ad hoc potential, as in the work to date $[6,7]$. In particular, one could consider an (Einstein frame) action such as

$$
S=\int \mathrm{d}^{D} x \sqrt{-g}\left\{\frac{\mathcal{R}}{4 \kappa^{2}}-\frac{1}{D-2} g^{a b} \partial_{a} \phi \partial_{b} \phi-\mathcal{V}(\phi)-\frac{1}{4} \exp \left(\frac{-4 \mathrm{~g}_{0} \kappa \phi}{D-2}\right) F_{a b} F^{a b}\right\},
$$

which includes gravity, an abelian gauge field and the dilaton, $\phi$, with a non-trivial dilaton potential $\mathcal{V}(\phi)$ of the form

$$
\mathcal{V}=\mathcal{V}_{\exp }+\mathcal{V}_{\text {susy }}
$$

where

$$
\mathcal{V}_{\exp }=\frac{\Lambda}{2 \kappa^{2}} \exp \left(\frac{-4 \mathrm{~g}_{1} \kappa \phi}{D-2}\right)
$$

\footnotetext{
$\ddagger$ A fascinating discussion of the observational consequences of a very weakly coupled massless dilaton is given in [5].

$\S$ Gregory and Harvey also considered a potential of the form $\mathcal{V}=2 m^{2} \cosh ^{2} \phi$.
} 
and

$$
\mathcal{V}_{\text {susy }}=\frac{1}{4 \kappa^{2}} \exp \left[-a e^{-2 \kappa \phi}\right]\left\{A e^{2 \kappa \phi}+B+C e^{-2 \kappa \phi}\right\}
$$

Here $\mathrm{g}_{0}, \mathrm{~g}_{1}, \Lambda, a, A, B$ and $C$ are constants and $\kappa^{2}=4 \pi G$ is the $D$-dimensional gravitational constant. Eqns. (1.1)-(1.2) are somewhat more general than is demanded by string theory. However, if we set $g_{0}=1$ we obtain the standard tree-level coupling between the dilaton and the electromagnetic field, while setting $\mathrm{g}_{1}=-1, \Lambda=\left(D_{\text {crit }}-D_{\text {eff }}\right) /\left(3 \alpha^{\prime}\right)$, in the Liouvilletype term (1.2) yields the case of a potential corresponding to a central charge deficit. The term (1.3), on the other hand, is the type of potential which arises in four dimensions from supersymmetry breaking via gaugino condensation in the hidden sector of the string theory [8]. Potentials of the form (1.2) and (1.3) have been widely studied in string cosmologies [9], but to date the only investigations of static spherically symmetric solutions involving such terms have been restricted to the case of uncharged solutions [10-14]. Maki and Shiraishi [15] have recently derived non-static Kastor-Traschen type [16] cosmological multi black hole solutions for the action (1.1)-(1.2). However, such solutions were only obtained for certain special values of the constants $g_{0}, g_{1}$ and of the time-dependent coupling in the dilaton cosmological scale factor.

In many respects the action (1.1) is still over-simplified because it neglects the possible contribution of additional scalar fields, such as the string moduli which correspond to the extra dimensions of spacetime after dimensional reduction. Static spherically symmetric solutions involving both moduli and a dilaton have been discussed recently by Cadoni and Mignemi [17], and by Cvetič and Tseytlin [18], but in the absence of a potential. The introduction of a potential greatly complicates the situation, however, as is well demonstrated by the case of the quadratic potential, where a complete integration proved impossible even numerically [7].

Given the inherent difficulties involved in studies of models with non-trivial potentials, the present paper is intended only as a first step: we will not study the problem posed by eqns. (1.1)-(1.3) in full, but will restrict ourselves solely to the case of a Liouville-type potential $\mathcal{V}=\mathcal{V}_{\exp }$. It is our hope that a further refinement of the approach discussed here can be applied to the more difficult case when a supersymmetry-breaking potential of the type (1.3) is also included. We are of course most interested in the case $D=4$, but will leave $D$ arbitrary, (with the only requirement that $D>2$ ), as this does not involve many extra complications. Furthermore, we will also leave the dilaton coupling to the electromagnetic field arbitrary, rather than immediately specialising to the string case $\left(g_{0}=1\right)$.

I The particular potential given in (1.3) is relevant for one gaugino condensation. 
We will show in the particular case of an exponential potential (1.2) that no static spherically symmetric asymptotically flat charged black hole solutions exist. Furthermore, no static spherically symmetric asymptotically de Sitter (or anti-de Sitter) solutions exist either, except in the special case $g_{1}=0$ when the potential is simply a cosmological constant. (The $\mathrm{g}_{1}=0$ model has recently been studied by Okai [19].) Our result concerning the potential (1.2) is of course more in the line with the intuition provided by the scalar no hair theorems [20-22], rather than with the dilaton black hole solutions [1] which avoid the no hair theorems through the coupling between the dilaton and electromagnetic fields, with the result that the dilaton scalar charge depends on the other charges of theory [23] rather than being an independent "hair".

\section{The dynamical system}

We shall use the same approach here as has been used by S. Mignemi and one of us [1012] to study uncharged static spherically symmetric solutions in models of gravity involving a scalar field with non-trivial potentials. Such an analysis is useful for deriving "no hair" results in circumstances in which some assumptions used in the standard no hair proofs do not apply. We recall, for example, that Bekenstein's proof [21] of the scalar no hair theorem for static black holes can be easily generalised to any convex potential [22], (i.e., for any $\mathcal{V}(\phi)$ for which $\frac{\mathrm{d}^{2} \mathcal{V}}{\mathrm{d} \phi^{2}} \geq 0$ for all $\phi$ ), by simply multiplying the appropriate scalar field equation by $\frac{\mathrm{d} \mathcal{V}}{\mathrm{d} \phi}$, rather than by $\phi$, before carrying out the appropriate integration. In [10], however, we derived the equivalent of a no hair result for the potential $(1.2)^{*}$ without any restriction on the sign of $\Lambda$. (For $\Lambda>0(\Lambda<0)$ the potential (1.2) is convex (concave).)

In [11] and [12] the approach of [10] was extended to more general potentials. Although the most general potential we have considered is an arbitrary finite sum of exponential terms

$$
\mathcal{V}(\phi)=\frac{-1}{4 \kappa^{2}} \sum_{i=1}^{s} \lambda_{i} \exp \left(\frac{-4 \mathrm{~g}_{i} \kappa \phi}{D-2}\right)
$$

there are some aspects of the analysis of [12] which would appear to apply to arbitrary potentials. In particular, one may conjecture that:

\footnotetext{
* In [10] a particular $g_{1}$ appropriate to Kaluza-Klein theories with internal spaces of non-zero curvature was used, but the arbitrary $g_{1}$ case was included in [12].
} 
(i) If $\mathcal{V}$ is non-zero then asymptotically (anti)-de Sitter solutions exist if and only if

$$
\exists \phi_{0} \text { such that }\left.\frac{\mathrm{d} \mathcal{V}}{\mathrm{d} \phi}\right|_{\phi=\phi_{0}}=0 \text { and } \mathcal{V}\left(\phi_{0}\right) \neq 0
$$

The solutions are asymptotically de Sitter (anti-de Sitter) for $\mathcal{V}\left(\phi_{0}\right)>0\left(\mathcal{V}\left(\phi_{0}\right)<0\right)$.

(ii) If $\mathcal{V}$ is non-zero then asymptotically flat solutions exist if and only if

$$
\exists \phi_{0} \text { such that }\left.\frac{\mathrm{d} \mathcal{V}}{\mathrm{d} \phi}\right|_{\phi=\phi_{0}}=0 \text { and } \mathcal{V}\left(\phi_{0}\right)=0
$$

The fact that trivial solutions exist under both these circumstances is pretty obvious: if (2.2) holds then the Schwarzschild-(anti)-de Sitter solutions with constant dilaton, $\phi=\phi_{0}$, are solutions; while if (2.3) is satisfied then the Schwarzschild solution with constant dilaton, $\phi=\phi_{0}$, is a solution. Any particular potential may have many such solutions, depending on the number of different turning points.

It is not difficult to see that any non-trivial solutions with the appropriate asymptotic form, and with a scalar field which is "physically well-behaved", namely at worst $\phi \sim$ const. at spatial infinity, must also satisfy (2.2) or (2.3) if all the fields have regular Taylor expansions at spatial infinity. This can in fact be seen by direct inspection of the field equations written in terms of a conventional radial coordinate. In particular, consider coordinates of the type used by Garfinkle, Horowitz and Strominger [2]:

$$
\mathrm{d} s^{2}=-e^{2 u} \mathrm{~d} t^{2}+e^{-2 u} \mathrm{~d} r^{2}+R^{2} \mathrm{~d} \Omega_{D-2}^{2},
$$

where $u=u(r)$ and $R=R(r)$. We will henceforth use units in which $\kappa=1$. The field equations obtained from variation of $(1.1)$ for a general potential $\mathcal{V}(\phi)$ are then given by

$$
\begin{aligned}
& \square \phi=\frac{1}{2}(D-2) \frac{\mathrm{d} \mathcal{V}}{\mathrm{d} \phi}-\frac{1}{2} \mathrm{~g}_{0} \exp \left(\frac{-4 \mathrm{~g}_{0} \phi}{D-2}\right) F_{a b} F^{a b}, \\
& \partial_{a}\left[\sqrt{-g} \exp \left(\frac{-4 \mathrm{~g}_{0} \phi}{D-2}\right) F^{a b}\right]=0, \\
& \mathcal{R}_{a b}=\frac{4}{D-2}\left[\partial_{a} \phi \partial_{b} \phi+g_{a b} \mathcal{V}\right]+2 \exp \left(\frac{-4 \mathrm{~g}_{0} \phi}{D-2}\right)\left[F_{a c} F_{b}{ }^{c}-\frac{1}{2(D-2)} g_{a b} F_{c d} F^{c d}\right],
\end{aligned}
$$


If we choose $\mathbf{F}$ to be the field of an isolated electric charge,

$$
\mathbf{F}=\exp \left(\frac{4 \mathrm{~g}_{0} \phi}{D-2}\right) \frac{Q}{R^{D-2}} \mathrm{~d} t \wedge \mathrm{d} r
$$

then the field equations with metric ansatz (2.4) are

$$
\begin{aligned}
\frac{1}{R^{D-2}} \frac{\mathrm{d}}{\mathrm{d} r}\left[R^{D-2} e^{2 u} \frac{\mathrm{d} \phi}{\mathrm{d} r}\right] & =\frac{1}{2}(D-2) \frac{\mathrm{d} \mathcal{V}}{\mathrm{d} \phi}+\mathrm{g}_{0} \exp \left(\frac{4 \mathrm{~g}_{0} \phi}{D-2}\right) \frac{Q^{2}}{R^{2(D-2)}} \\
\frac{1}{R} \frac{\mathrm{d}^{2} R}{\mathrm{~d} r^{2}} & =-\frac{4}{(D-2)^{2}}\left(\frac{\mathrm{d} \phi}{\mathrm{d} r}\right)^{2}, \\
\frac{1}{R^{D-2}} \frac{\mathrm{d}}{\mathrm{d} r}\left[e^{2 u} \frac{\mathrm{d}}{\mathrm{d} r}\left(R^{D-2}\right)\right] & =(D-2)(D-3) \frac{1}{R^{2}}-4 \mathcal{V}-2 \exp \left(\frac{4 \mathrm{~g}_{0} \phi}{D-2}\right) \frac{Q^{2}}{R^{2(D-2)}},
\end{aligned}
$$

together with one further equation which depends on the others by virtue of the Bianchi identity. Although we have assumed an electric field here, the solutions for a purely magnetic field are readily obtainable once the solutions for the system (2.6) are known since the field equations for the magnetic case can be obtained from (2.6) by making the replacement $\mathrm{g}_{0} \rightarrow-\mathrm{g}_{0}$ and $Q \rightarrow P$, where $P$ is the magnetic monopole charge.

If we now make the expansions

$$
\begin{aligned}
e^{2 u} & =\frac{-2 \Lambda r^{2}}{(D-1)(D-2)}+u_{-1} r+u_{0}+\frac{u_{1}}{r}+\frac{u_{2}}{r^{2}}+\ldots, \\
R & =r+R_{0}+\frac{R_{1}}{r}+\frac{R_{2}}{r^{2}}+\ldots, \\
\phi & =\phi_{0}+\frac{\phi_{1}}{r}+\frac{\phi_{2}}{r^{2}}+\ldots,
\end{aligned}
$$

at spatial infinity, assuming the solutions to be asymptotically (anti)-de Sitter or asymptotically flat depending on the value of $\Lambda$, then substitution of (2.7) in (2.6) yields the result

$$
\left.\frac{\mathrm{d} \mathcal{V}}{\mathrm{d} \phi}\right|_{\phi=\phi_{0}}=0, \quad \Lambda=2 \mathcal{V}\left(\phi_{0}\right)
$$

from the lowest order terms. Thus in general it is necessary for the potential to have a turning point at $\phi=\phi_{0}$ for solutions with asymptotic expansions (2.7) to exist, and such solutions will be asymptotically flat, de Sitter or anti-de Sitter if $\mathcal{V}\left(\phi_{0}\right)=0, \mathcal{V}\left(\phi_{0}\right)>0$ or $\mathcal{V}\left(\phi_{0}\right)<0$ respectively. Such solutions are consequently ruled out for the Liouville-type potential (1.2), except in the special case of a cosmological constant $\left(g_{1}=0\right)$ when $\frac{d \mathcal{V}}{d \phi}=0$ identically. 
If we make no assumptions about the existence of regular series expansions at spatial infinity then the proof of necessity in (2.2) and (2.3) is much less trivial. Indeed, in the context of models of gravity corresponding to the low-energy limit of string theory one can conceive of instances in which power series expansions of the form (2.7) would not apply. In particular, although asymptotics with $\phi \rightarrow-\infty$ at spatial infinity would be disastrous in conventional field theories, in the case of string theory all couplings between the dilaton and matter fields involve powers of $e^{2 \phi}$, so provided the dilaton energy-momentum tensor is well-behaved at spatial infinity one would expect the weak-coupling limit $\phi \rightarrow-\infty$ to be physically admissable. It is under such circumstances that the approach of [10-12] becomes useful: we reformulate the field equations in terms of a first order autonomous system of ordinary differential equations. Typically one finds that the full phase space has various subspaces, one of which corresponds to the system with no potential and which contains critical points at the phase space infinity that correspond physically to an asymptotically flat region. If such critical points are not endpoints for integral curves which lie outside of the subspace with $\mathcal{V}(\phi) \equiv 0$, and if no other critical points correspond to an asymptotically flat region, then a "no hair" result is obtained. The precise global properties of all solutions of interest are often readily obtained.

We will now apply this approach to the problem posed by the action (1.1)-(1.2). In order to obtain an autonomous system one must use the radial coordinate of Gibbons and Maeda [1], defined by

$$
\mathrm{d} \xi=\frac{e^{-2 u} \mathrm{~d} r}{R^{D-2}}
$$

in terms of the previous radial coordinate $r$. One further modification is necessary for the phase space analysis, namely to replace the $(D-2)$-sphere of the spatial section by a more general $(D-2)$-dimensional Einstein space, so that the full metric is given by

$$
\mathrm{d} s^{2}=\varepsilon e^{2 u}\left[-\mathrm{d} t^{2}+R^{2(D-2)} \mathrm{d} \xi^{2}\right]+R^{2} g_{i j} \mathrm{~d} x^{i} \mathrm{~d} x^{j}
$$

where now $u=u(\xi), R=R(\xi)$,

$$
\mathcal{R}_{i j}=(D-3) \bar{\lambda} g_{i j}, \quad i=1, \ldots, D-2,
$$

and $\varepsilon= \pm 1$. Of course, we are primarily interested in the case when $\bar{\lambda}=1$ and $g_{i j}$ is the standard metric for a $(D-2)$-sphere. However, the $\bar{\lambda}=0$ surface forms an important boundary in the phase space, with various critical points lying there, and thus we must leave $\bar{\lambda}$ arbitrary for the analysis. We have included a factor $\varepsilon= \pm 1$ explicitly in (2.9), as this will 
allow for the inclusion of critical points both in the region in which the Killing vector $\partial / \partial t$ is timelike $(\varepsilon=+1)$, and the region in which $\partial / \partial t$ is spacelike $(\varepsilon=-1)$, in the analysis below.

In terms of the coordinates (2.9) the field equations are

$$
\begin{gathered}
\phi^{\prime \prime}=-\mathrm{g}_{1} \varepsilon \Lambda e^{2 \chi}+\mathrm{g}_{0} \varepsilon Q^{2} e^{2 \eta} \\
\zeta^{\prime \prime}=(D-3)^{2} \varepsilon \bar{\lambda} e^{2 \zeta}-2 \varepsilon \Lambda e^{2 \chi} \\
u^{\prime \prime}=\frac{-2}{D-2} \varepsilon \Lambda e^{2 \chi}+2\left(\frac{D-3}{D-2}\right) \varepsilon Q^{2} e^{2 \eta} \\
\left(\frac{D-2}{D-3}\right)\left[\zeta^{\prime 2}-u^{\prime 2}\right]-\frac{4}{(D-2)} \phi^{\prime 2}-(D-2)(D-3) \varepsilon \bar{\lambda} e^{2 \zeta}+2 \varepsilon \Lambda e^{2 \chi}+\varepsilon Q^{2} e^{2 \eta}=0
\end{gathered}
$$

where

$$
\zeta \equiv u+(D-3) \ln R, \quad \eta \equiv u+\frac{2 \mathrm{~g}_{0} \phi}{D-2}, \quad \chi \equiv u+(D-2) \ln R-\frac{2 \mathrm{~g}_{1} \phi}{D-2} .
$$

If we now define

$$
X=\zeta^{\prime}, \quad Y=\eta^{\prime}, \quad Z=\sqrt{\frac{2}{D-2}} e^{\eta}, \quad V=\chi^{\prime}, \quad W=\sqrt{\frac{2}{D-2}} e^{\chi},
$$

then the constraint equation (2.13) may be used to eliminate the terms involving $\bar{\lambda}$ from the field equations (2.10)-(2.12), yielding the following first order autonomous dynamical system:

$$
\begin{aligned}
X^{\prime} & =(D-3) \varepsilon Q^{2} Z^{2}-\varepsilon \Lambda W^{2}-(D-3) \mathcal{P} \\
Y^{\prime} & =\left(D-3+\mathrm{g}_{0}^{2}\right) \varepsilon Q^{2} Z^{2}-\left(1+\mathrm{g}_{0} \mathrm{~g}_{1}\right) \varepsilon \Lambda W^{2} \\
V^{\prime} & =\left(D-3-\mathrm{g}_{0} \mathrm{~g}_{1}\right) \varepsilon Q^{2} Z^{2}+\left(\mathrm{g}_{1}^{2}-1\right) \varepsilon \Lambda W^{2}-(D-2) \mathcal{P} \\
Z^{\prime} & =Y Z \\
W^{\prime} & =V W
\end{aligned}
$$

where

$$
\begin{aligned}
& \mathcal{P} \equiv \frac{1}{\left[(D-3) \mathrm{g}_{1}-\mathrm{g}_{0}\right]^{2}}\{ {\left[(D-1) \mathrm{g}_{0}^{2}+(D-2)^{2}+2 \mathrm{~g}_{0} \mathrm{~g}_{1}-(D-3) \mathrm{g}_{1}^{2}\right] X^{2} } \\
&+\left[1+(D-3) \mathrm{g}_{1}{ }^{2}\right] Y^{2}+\left(D-3+\mathrm{g}_{0}{ }^{2}\right) V[(D-3) V-2(D-2) X] \\
&\left.+2\left(1+\mathrm{g}_{0} \mathrm{~g}_{1}\right) Y[(D-3) V-(D-2) X]\right\}
\end{aligned}
$$


and $g_{0} \neq(D-3) g_{1}$. (We will consider the particular case $g_{0}=(D-3) g_{1}$ at a later stage).

Our aim is to analyse the phase space for this system of equations. Since the metric and dilaton field are related to the functions $X, Y, V, Z$ and $W$ of the 5-dimensional phase space, they are necessarily regular at all points of the integrals curves apart from critical points. Consequently, in order to determine the global properties of all solutions - namely the structure of their singularities, horizons and asymptotic regions - it suffices to study the properties of the solutions at critical points of the phase space. Further careful analysis is required in order to determine which critical points are connected to which other ones by integral curves, thus determining the different possibilities for spacetime structure.

Although the space is 5-dimensional we have some hope of analysing it due to various symmetries. Equations (2.19) and (2.20) ensure that trajectories cannot cross either the $W=0$ or $Z=0$ subspaces, which correspond physically to $\Lambda=0$ and $Q=0$ respectively. Thus we may restrict our attention to $Z \geq 0$ and $W \geq 0$ without loss of generality. The hyperboloid defined by $\bar{\lambda}=0$, or equivalently from (2.13)

$$
\mathcal{P}-\varepsilon Q^{2} Z^{2}-\varepsilon \Lambda W^{2}=0
$$

similarly forms a surface which trajectories cannot cross. It partitions the phase space into the two physically distinct regions with $\bar{\lambda}>0$ and $\bar{\lambda}<0$.

\section{The $W=0$ and $Z=0$ subspaces}

If $W=0$ or $Z=0$, which corresponds physically to $\Lambda=0$ and $Q=0$ respectively, then one equation (either (2.20) or (2.19)) drops out and the phase space becomes 4-dimensional. In both cases, however, one direction in the 4-dimensional subspace is "trivial" as a further degree of freedom can be integrated out with a linear dependence on two of the other dimensions. In particular, if $W=0$ then

$$
V=\left(\frac{D-2}{D-3}\right) X-\left(\frac{1+\mathrm{g}_{0} \mathrm{~g}_{1}}{D-3+\mathrm{g}_{0}^{2}}\right) Y+\left(\frac{(D-3) \mathrm{g}_{1}-\mathrm{g}_{0}}{D-3}\right) c_{0}
$$

while if $Z=0$ then

$$
Y=\frac{\left(1+\mathrm{g}_{0} \mathrm{~g}_{1}\right)[(D-2) X-(D-3) V]+\left[(D-3) \mathrm{g}_{1}-\mathrm{g}_{0}\right] c_{1}}{1+(D-3) \mathrm{g}_{1}{ }^{2}}
$$

where $c_{0}$ and $c_{1}$ are arbitrary constants. Of course, the $W=0$ (i.e., $\Lambda=0$ ), system can be integrated completely [1]. (See also Appendix B.) However, for our purposes here it is sufficient to stop with (3.1) and analyse the critical points. 
The only critical points at a finite distance from the origin in the full 5 -dimensional phase space have both $W=0$ and $Z=0$, and so are common to both subspaces. These critical points also have $\mathcal{P}=0$, and consequently by (2.22) it follows that they are on the $\bar{\lambda}=0$ surface also. From (2.21) it follows that these points are located at $X=X_{0}, Y=Y_{0}$, and $V=V_{0}$ where

$$
\begin{aligned}
& \left|X_{0}\right| \geq\left(D-3+\mathrm{g}_{0}^{2}\right)^{1 / 2}\left|c_{0}\right|, \\
& Y_{0}= \pm\left(\frac{D-3+\mathrm{g}_{0}^{2}}{D-3}\right)^{1 / 2}\left[X_{0}{ }^{2}-\left(D-3+\mathrm{g}_{0}{ }^{2}\right) c_{0}{ }^{2}\right]^{1 / 2}
\end{aligned}
$$

while $V_{0}$ is given by substituting (3.3) in (3.1).

Consider the $W=0(\Lambda=0)$ subspace. If the $V$-direction is parametrised as in (3.1) then by eliminating $V$ from the other equations we obtain an effective 3 -dimensional system:

$$
\begin{aligned}
X^{\prime} & =(D-3) \varepsilon Q^{2} Z^{2}+X^{2}-\left(\frac{D-3}{D-3+\mathrm{g}_{0}^{2}}\right) Y^{2}-\left(D-3+\mathrm{g}_{0}^{2}\right) c_{0}^{2} \\
Y^{\prime} & =\left(D-3+\mathrm{g}_{0}{ }^{2}\right) \varepsilon Q^{2} Z^{2} \\
Z^{\prime} & =Y Z
\end{aligned}
$$

The integral curves which lie in the plane $Z=0$ are just the lines $Y=$ const. Such curves correspond physically to the spacetimes with $Q=0$ and $\Lambda=0$, and the general solution for the physically interesting $\bar{\lambda}=1$ case was given long ago by Buchdahl [24]. The exact solutions for all values of $\bar{\lambda}$ are given in [10] and [12]. For each value of $c_{0}$ the critical points (3.3) form a hyperbola in the $Z=0$ plane. From the analysis of [10-12] it follows that for each value of $c_{0}$ one critical point, namely the point with ${ }^{\star}$

$$
X_{0}=Y_{0}=-\left(D-3+\mathrm{g}_{0}^{2}\right) \frac{c_{0}}{\mathrm{~g}_{0}}, \quad \mathrm{~g}_{0} \neq 0
$$

corresponds to an horizon, $r \rightarrow r_{\mathcal{H}}$, while the remaining critical points correspond to a singularity at $r \rightarrow 0$. The trajectory with an endpoint at the horizon which lies completely in the $Z=0$ plane of course corresponds to the Schwarzschild solution and the constant $c_{0}$ is related to the Schwarzschild radius. (See e.g. Fig. 1 in [10].)

\footnotetext{
$\star$ The case $\mathrm{g}_{0}=0$, for which the $W=0$ subspace just represents the standard Reissner-Nordström solution can readily be treated by a separate analysis. However, this will not concern us here.
} 
An analysis of small perturbations about the critical points $X=X_{0}, Y=Y_{0}, Z=$ 0 in the 3 -dimensional subspace yields the eigenvalues $0,2 X_{0}, Y_{0}$. The zero eigenvalue corresponds to the degeneracy in the $Y$ direction on the $Z=0$ plane. Each critical point in the first and third quadrants is the endpoint of a 2-dimensional bunch of trajectories in the 3-dimensional space, while those in the second and fourth quadrants are saddle points with respect to trajectories out of the $Z=0$ plane.

The critical points which lie on the sphere at infinity in the effective 3-dimensional phase space may also be found by standard means. These points may be classified as follows:

$$
\begin{array}{lll}
J_{1,2}: \quad X=0, Y= \pm \infty, Z=\frac{Y}{\sqrt{\varepsilon Q^{2}\left(D-3+\mathrm{g}_{0}^{2}\right)}}, & \Rightarrow \mathcal{P}=\frac{Y^{2}}{D-3+\mathrm{g}_{0}^{2}}, \\
K_{1,2}: \quad X= \pm \infty, Y=X, Z=\frac{X}{\sqrt{\varepsilon Q^{2}\left(D-3+\mathrm{g}_{0}^{2}\right)}}, & \Rightarrow \mathcal{P}=\frac{-\mathrm{g}_{0}^{2} X^{2}}{(D-3)\left(D-3+\mathrm{g}_{0}^{2}\right)}, \\
L_{1-4}: \quad X= \pm \infty, Y= \pm X \sqrt{\frac{D-3+\mathrm{g}_{0}^{2}}{D-3}, Z=0,} \quad \Rightarrow \quad \mathcal{P}=0, \\
M_{1,2}: \quad X= \pm \infty, Y=0, Z=0, \quad \Rightarrow \mathcal{P}=-X^{2} /(D-3),
\end{array}
$$

The points $L_{1-4}$ of course correspond to the endpoints of the one-parameter family of critical points given by (3.3), while the points $M_{1,2}$, are labelled here so as to correspond to the critical points at infinity with the same physical properties as in [10-12]. Since the phase space here describes a system which is physically different to the models discussed in [10-12], the points $J_{1,2}$ and $K_{1,2}$ have no direct correspondence to cases considered there. In Fig. 1 we plot the surface of the sphere at infinity in terms of the coordinates $\theta_{1}$ and $\phi_{1}$ defined by

$$
X=\bar{\rho}(1-\bar{\rho})^{-1} \sin \theta_{1} \cos \phi_{1}, Y=\bar{\rho}(1-\bar{\rho})^{-1} \sin \theta_{1} \sin \phi_{1}, Z=\bar{\rho}(1-\bar{\rho})^{-1} \cos \theta_{1}
$$

in the limit $\bar{\rho} \rightarrow 1$. We will postpone the discussion of the properties of solutions approaching these points until the next section.

Now consider the $Z=0(Q=0)$ subspace. If the $Y$-direction is parametrised as in (3.1) then by eliminating $Y$ from the other equations we obtain an effective 3-dimensional system:

$$
\begin{aligned}
X^{\prime} & =-\varepsilon \Lambda W^{2}-(D-3) \mathcal{P} \\
Y^{\prime} & =\left(\mathrm{g}_{1}{ }^{2}-1\right) \varepsilon \Lambda W^{2}-(D-2) \mathcal{P} \\
W^{\prime} & =V W
\end{aligned}
$$


with

$$
\mathcal{P}=\frac{1}{1+(D-3) \mathrm{g}_{1}^{2}}\left[\left(D-1-\mathrm{g}_{1}^{2}\right) X^{2}-2(D-2) X V+(D-3) V^{2}+c_{1}^{2}\right]
$$

in this case. This system, which is physically equivalent to Einstein gravity coupled to a scalar field with a single exponential potential is of course precisely one of the systems that have already been studied in [10-12], and the properties of the solutions are identical. In addition to the critical points common to the $W=0$ and $Z=0$ given above the following additional critical points are found:

$$
\begin{aligned}
& L_{5-8}: \quad X= \pm \infty, V=\frac{(D-2) \pm \sqrt{1+(D-3) \mathrm{g}_{1}^{2}}}{D-3}, W=0, \quad \Rightarrow \quad \mathcal{P}=0, \\
& N_{1,2}: X= \pm \infty, V=\left(\frac{D-1-\mathrm{g}_{1}^{2}}{D-2}\right) X, W=X \sqrt{\frac{D-1-\mathrm{g}_{1}^{2}}{-\varepsilon \Lambda}}, \\
& P_{1,2}: \quad X= \pm \infty, V=X, W=\frac{\mathcal{P}=\frac{\left(\mathrm{g}_{1}^{2}-(D-1)\right) X^{2}}{(D-2)^{2}} .}{\sqrt{-\varepsilon \Lambda\left(1+(D-3) \mathrm{g}_{1}^{2}\right)}}, \quad \Rightarrow \quad \mathcal{P}=\frac{-\mathrm{g}_{1}^{2} X^{2}}{1+(D-3) \mathrm{g}_{1}^{2}},
\end{aligned}
$$

The points $L_{5-6}, N_{1,2}$ and $P_{1,2}$ have been labelled here in precisely the same fashion as in $[12]$.

\section{The 5-dimensional phase space}

It is not extremely difficult to piece together the structure of the integral curves in the full 5-dimensional phase space $\{X, Y, Z, V, W\}$ given the existence of the various symmetries and special subspaces discussed above. As we have already noted all critical points in the 5 -dimensional phase space which lie at a finite distance from the origin are confined to the $W=0, Z=0$ subspace, and this leads to great simplifications. Similarly most of the critical points at the phase space infinity are those obtained in the previous section; the only additional critical points are found to be the one parameter family $L(y)$ :

$$
X= \pm \infty, Y=y X, V=v X, Z=0, W=0,
$$


where

$$
-\left[\frac{D-3+\mathrm{g}_{0}^{2}}{(D-3)}\right]^{1 / 2} \leq y \leq\left[\frac{D-3+\mathrm{g}_{0}^{2}}{(D-3)}\right]^{1 / 2}
$$

and $^{\star}$

$v=\frac{(D-2)\left[D-3+\mathrm{g}_{0}^{2}\right]-(D-3)\left(1+\mathrm{g}_{0} \mathrm{~g}_{1}\right) \pm\left[(D-3) \mathrm{g}_{1}-\mathrm{g}_{0}\right] \sqrt{\mathrm{g}_{0}^{2}+(D-3)\left(1-y^{2}\right)}}{(D-3)\left[D-3+\mathrm{g}_{0}^{2}\right]}$,

together with the isolated points

$$
\begin{array}{r}
S_{1,2}: \quad X= \pm \infty, Y=X, V=X, \\
W=X \sqrt{\frac{\mathrm{g}_{0}}{\varepsilon \Lambda\left((D-3) \mathrm{g}_{1}-\mathrm{g}_{0}\right)}}, Z=X \sqrt{\frac{\mathrm{g}_{1}}{\varepsilon Q^{2}\left((D-3) \mathrm{g}_{1}-\mathrm{g}_{0}\right)}}, \quad \Rightarrow \quad \mathcal{P}=0, \\
T_{1,2}: X= \pm \infty, Y=V=X\left(\frac{\alpha_{1}}{\alpha_{2}}\right), W=X \sqrt{\frac{\alpha_{1}}{-\varepsilon \Lambda(D-2) \alpha_{2}}}, Z=X \sqrt{\frac{\alpha_{1} \alpha_{3}}{\varepsilon Q^{2} \alpha_{2}{ }^{2}}}, \\
\Rightarrow \mathcal{P}=\frac{-\left(\mathrm{g}_{0}+\mathrm{g}_{1}\right)^{2} \alpha_{1} X^{2}}{\alpha_{2}{ }^{2}},
\end{array}
$$

where

$$
\begin{aligned}
& \alpha_{1} \equiv(D-1) \mathrm{g}_{0}{ }^{2}+2 \mathrm{~g}_{0} \mathrm{~g}_{1}-(D-3) \mathrm{g}_{1}^{2}+(D-2)^{2} \\
& \alpha_{2} \equiv(D-2)\left[\mathrm{g}_{0}\left(\mathrm{~g}_{0}+\mathrm{g}_{1}\right)+(D-2)\right] \\
& \alpha_{3} \equiv(D-2)-\mathrm{g}_{1}\left(\mathrm{~g}_{0}+\mathrm{g}_{1}\right) .
\end{aligned}
$$

The points $L(y)$ represent the extension of points $L_{1-8}$ to the 1-parameter set of critical points which coincide with the intersection of the $\bar{\lambda}=0, Z=0, W=0$ surface and the sphere at infinity.

Following [10-12] we will specify the asymptotic behaviour of solutions by using the proper radius $R$ as the radial variable through coordinates

$$
\mathrm{d} s^{2}=-e^{2 u} \mathrm{~d} t^{2}+e^{2 v} \mathrm{~d} R^{2}+R^{2} g_{i j} \mathrm{~d} x^{i} \mathrm{~d} x^{j}
$$

where now $u=u(R)$ and $v=v(R)$, instead of coordinates (2.4) or (2.9). The properties of the solutions in the neighbourhood of the various critical points may be readily determined.

$\star$ Note: there is a factor $\left(g_{1}-g_{2}\right)$ missing from in front of the square root term in the corresponding equation (2.42) in [12]. 
One finds three possible behaviours for the proper radius $R$ : (i) $R \rightarrow 0$ corresponding to a central singularity as in Table 1 ; (i) $R \rightarrow \infty$, corresponding to an asymptotic region as in Table 2 ; or (ii) $R=$ const. which is true only in the case of the points $S_{1,2}$. Points $S_{1,2}$ have $\phi=$ constant also, and they correspond to the endpoints of Robinson-Bertotti type solutions as is demonstrated in Appendix A.

\begin{tabular}{|l|l|l|l|l|}
\hline & Values of constants & $e^{2 u}$ & $e^{2 v}$ & $e^{2 \phi}$ \\
\hline$J_{1,2}$ & $\varepsilon=+1, \bar{\lambda}=0$ & $R^{-2(D-3)}$ & $R^{2\left[\mathrm{~g}_{1}{ }^{2}+(D-3)\right]}$ & $R^{-(D-2) \mathrm{g}_{0}}$ \\
\hline$N_{1,2}$ & $\mathrm{~g}_{1}{ }^{2}>(D-1), \varepsilon \Lambda<0, \bar{\lambda}=0$ & $R^{2}$ & $R^{2\left(\mathrm{~g}_{1}{ }^{2}-1\right)}$ & $R^{(D-2) \mathrm{g}_{1}}$ \\
\hline
\end{tabular}

Table 1 Asymptotic form of solutions for trajectories approaching critical points at phase space infinity from within the sphere at infinity, in the case that $R \rightarrow 0$.

\begin{tabular}{|l|l|l|l|l|}
\hline & Values of constants & $e^{2 u}$ & $e^{2 v}$ & $e^{2 \phi}$ \\
\hline$K_{1,2}$ & $\varepsilon=+1, \bar{\lambda}>0$ & $R^{2(D-3)^{2} / \mathrm{g}_{0}{ }^{2}}$ & const. & $R^{(D-2)(D-3) / \mathrm{g}_{0}}$ \\
\hline$M_{1,2}$ & $\varepsilon \bar{\lambda}>0$ & const. & const. & const. \\
\hline$N_{1,2}$ & $\mathrm{~g}_{1}{ }^{2}<(D-1), \varepsilon \Lambda<0, \bar{\lambda}=0$ & $R^{2}$ & $R^{2\left(\mathrm{~g}_{1}{ }^{2}-1\right)}$ & $R^{(D-2) \mathrm{g}_{1}}$ \\
\hline$P_{1,2}$ & $\varepsilon \Lambda<0, \operatorname{sign} \bar{\lambda}=\operatorname{sign} \varepsilon\left(\mathrm{g}_{1}{ }^{2}-1\right)$ & $R^{2 / \mathrm{g}_{1}{ }^{2}}$ & const. & $R^{(D-2) / \mathrm{g}_{1}}$ \\
\hline$T_{1,2}$ & $\varepsilon \Lambda \alpha_{1} / \alpha_{2}<0, \bar{\lambda}=0, \varepsilon \alpha_{1} \alpha_{3}>0$ & $R^{2 \beta_{1}}$ & $R^{2 \beta_{2}}$ & $R^{(D-2)^{2} /\left(\mathrm{g}_{0}+\mathrm{g}_{1}\right)}$ \\
\hline
\end{tabular}

Table 2 Asymptotic form of solutions for trajectories approaching critical points at phase space infinity from within the sphere at infinity, in the case that $R \rightarrow \infty$.

Here $\beta_{1}=1+(D-2)\left[(D-2)-\mathrm{g}_{1}\left(\mathrm{~g}_{0}+\mathrm{g}_{1}\right)\right] /\left(\mathrm{g}_{0}+\mathrm{g}_{1}\right)^{2}$ and $\beta_{2}=1-(D-2) \mathrm{g}_{1} /\left(\mathrm{g}_{0}+\mathrm{g}_{1}\right)$.

The only critical points which correspond to an asymptotically flat region are $M_{1,2}$, and as anticipated from our earlier analysis no critical points in Table 1 have (anti)-de Sitter asymptotics, except in the special case $\mathrm{g}_{1}=0$ when the points $N_{1,2}$ are (anti)-de Sitter. In order to determine the nature of the set of trajectories which have endpoints at the various 


\begin{tabular}{|c|c|}
\hline & Eigenvalues (with degeneracies) \\
\hline$K_{1}$ & $-2 ;-1,(2) ; 1 ; \frac{\mathrm{g}_{0}\left[\mathrm{~g}_{0}-(D-3) \mathrm{g}_{1}\right]}{(D-3)\left[D-3+\mathrm{g}_{0}^{2}\right]}$. \\
\hline$L(y)$ & $0,(2) ; 2 ; y ; v .^{*}$ \\
\hline$M_{1}$ & $-1,(4) ; \frac{1}{D-3}$. \\
\hline$N_{1}$ & $\frac{-\left(D-1-\mathrm{g}_{1}^{2}\right)}{D-2}(3) ; \frac{2}{D-2}\left(\mathrm{~g}_{1}^{2}-1\right) ; \frac{\mathrm{g}_{1}}{D-2}\left(\mathrm{~g}_{0}+\mathrm{g}_{1}\right)-1$. \\
\hline$P_{1}$ & $-1,(2) ; \frac{-\mathrm{g}_{1}\left[(D-3) \mathrm{g}_{1}-\mathrm{g}_{0}\right]}{1+(D-3) \mathrm{g}_{1}^{2}} ; \frac{-1}{2}\left[1 \pm \sqrt{\frac{9+(D-11) \mathrm{g}_{1}^{2}}{1+(D-3) \mathrm{g}_{1}^{2}}}\right.$ \\
\hline$S_{1}$ & $-2 ;-1 ; 1 ; \frac{1}{2}\left\{1 \pm \sqrt{1+\frac{8 \mathrm{~g}_{0} \mathrm{~g}_{1}\left(\mathrm{~g}_{0}+\mathrm{g}_{1}\right)}{(D-3) \mathrm{g}_{1}-\mathrm{g}_{0}}}\right.$ \\
\hline$T_{1}$ & $\begin{array}{l}-\left(\frac{\alpha_{1}}{\alpha_{2}}\right),(2) ; \frac{2}{\alpha_{2}}\left(\mathrm{~g}_{0}+\mathrm{g}_{1}\right)\left((D-3) \mathrm{g}_{1}-\mathrm{g}_{0}\right) ; \\
\frac{\sqrt{\alpha_{1}}}{2 \alpha_{2}}\left\{-\sqrt{\alpha_{1}} \pm \sqrt{9(D-2)\left[\mathrm{g}_{0}^{2}-\mathrm{g}_{1}^{2}+(D-2)\right]+\left(\mathrm{g}_{0}+\mathrm{g}_{1}\right)^{2}\left(1-8 \mathrm{~g}_{0} \mathrm{~g}_{1}\right)}\right.\end{array}$ \\
\hline
\end{tabular}

Table 3 Eigenvalues of critical points at phase space infinity. The eigenvalues for small perturbations which are degenerate have the degeneracy listed in brackets.

* The values of $y$ and $v$ listed are defined by (4.2) and (4.3).

critical points it only remains to find the eigenvalues spectrum of linearised perturbations. These results are given in Table 3. 


\section{The structure of the phase space}

For $\Lambda>0$ and $\varepsilon=1$ the only critical points at phase space infinity for which $R \rightarrow \infty$ are $K_{1,2}$ and $M_{1,2}$, which both lie in the $W=0$ subspace (corresponding to $\Lambda=0$ ). The only trajectories approaching $M_{1}$ are the asymptotically flat solutions lying entirely in the $W=0$ subspace, which of course include the familiar Gibbons-Maeda dilaton black-hole solutions. In general, trajectories which approach the points $K_{1,2}$ lie entirely within the $W=0$ subspace. However, if $\mathrm{g}_{0}^{2}>(D-3) \mathrm{g}_{0} \mathrm{~g}_{1}$ then there will be trajectories which approach this critical point from outside the $W=0$ subspace.

On the face of it trajectories approaching the critical points $K_{1,2}$ are potentially of interest, particularly for those trajectories which correspond to the weak coupling limit $e^{2 \phi} \rightarrow 0$. Indeed, one finds that the curvature invariants of spacetimes asymptotic to $K_{1,2}$ do have suggestive properties. To be specific, in the case $D=4, \mathrm{~g}_{0}=-1$ all the components of the Riemann tensor in an orthonormal frame go to zero as $1 / R^{2}$ as $R \rightarrow \infty$, and curvature invariants go to zero as $1 / R^{4}$. Nevertheless, although the $x^{i}=$ const. section resembles a 2dimensional Rindler spacetime as $R \rightarrow \infty$, the global structure is such that the 4-dimensional spacetime is not asymptotically flat. The explicit solutions which approach $K_{1}$ from within the $W=0$ subspace are found in Appendix B for arbitrary $D$ and $g_{0}$. It is seen that all of these correspond to naked singularities.

There are no other critical points with $R \rightarrow \infty$ for $\Lambda>0$. All the trajectories for this system which enter the $\varepsilon=1$ region either end at: $M_{1,2}, K_{1,2}$, the Robinson-Bertotti type points $S_{1,2}$, a central singularity, or at an horizon. The Robinson-Bertotti type points exist if

$$
\begin{array}{ll} 
& \varepsilon \Lambda /\left[\mathrm{g}_{0}\left((D-3) \mathrm{g}_{1}-\mathrm{g}_{0}\right)\right]>0, \\
& \varepsilon /\left[\mathrm{g}_{1}\left((D-3) \mathrm{g}_{1}-\mathrm{g}_{0}\right)\right]>0, \\
\text { and } \quad & \operatorname{sign} \bar{\lambda}=\operatorname{sign} \varepsilon\left(\mathrm{g}_{0}+\mathrm{g}_{1}\right) /\left[\mathrm{g}_{0}\left((D-3) \mathrm{g}_{1}-\mathrm{g}_{0}\right)\right] .
\end{array}
$$

The point $S_{1}$ attracts a 3 -dimensional bunch of trajectories. These are given explicitly in Appendix A.

If a trajectory has an endpoint at an horizon it will pass into the $\varepsilon<0$ region and its asymptotics will be found by considering the $\varepsilon<0$ critical points. So we will now consider the various possibilities for such trajectories. We note that it is the product of $\varepsilon$ and $\Lambda$ that is important. Thus all the remarks below about the critical points with $\varepsilon=-1$ and $\Lambda>0$ also apply to the case for which $\varepsilon=1$ and $\Lambda<0$.

As in the case of the models discussed in [12], the structure of the phase space is quite distinct acording whether $\mathrm{g}_{1}{ }^{2}<(D-1)$ or $\mathrm{g}_{1}{ }^{2}>(D-1)$. For $\mathrm{g}_{1}{ }^{2}<(D-1)$ the critical 
point $N_{1}$, lies at phase space infinity and for small $\mathrm{g}_{1}$ it attracts a 5-dimensional bunch of trajectories. For $\mathrm{g}_{1}=0$ trajectories approaching $N_{1}$ are asymptotically de Sitter. While we cannot prove explicitly that analogues of Reissner-Nordström-de Sitter solutions exist in the case $g_{1}=0$, the fact that the point $N_{1}$ is a 5 -dimensional attractor combined with the fact that the $Z=0$ subspace is known to contain trajectories with two horizons which end at the point $N_{1}$ - namely the Schwarzschild-de Sitter solutions - make it very likely that at least some perturbations away from the $Z=0$ subspace end at $N_{1}$ and have two horizons. As a check on these arguments numerical work is in progress and will be reported elsewhere ${ }^{\star}$ [25]. In a recent paper Okai [19] reaches similar conclusions, and shows that if such black hole solutions exist (for $g_{1}=0$ ) then there can be at most two horizons. At this stage the reader should also recall that we are not allowing $\mathrm{g}_{0}=0$, and hence the usual Reissner-Nordströmde Sitter solutions are not contained in our system. It is straightforward to repeat the entire analysis for the case $\mathrm{g}_{0}=0$ to obtain the standard results.

The points $P_{1,2}$ which lie in the $Z=0$ (i.e. $Q=0$ ) subspace have asymptotics identical to those of the points $K_{1,2}$ if one makes the replacement $\mathrm{g}_{0} \rightarrow \mathrm{g}_{1} /(D-3)$, so the above remarks about $K_{1,2}$ apply here as well. In fact, the case of the potential corresponding to a central charge deficit in 4 dimensions, $g_{1}=-1$, has the precise behaviour of the particular example discussed above. If we restrict our attention to the cases in which $P_{1,2}$ can be endpoints for trajectories for physical spacetimes with $\bar{\lambda}>1$ then we obtain the restriction that $\mathrm{g}_{1}{ }^{2} \geq 1$. The point $P_{1}$ is either a 3 -dimensional or 4-dimensional attractor depending on the relative signs of $g_{0}$ and $g_{1}$. For some regions of the parameter space there will be trajectories with endpoints there for non-zero $Q$.

Finally, the asymptotics of the points $T_{1,2}$ are in general very complicated. It is easy to verify that there are no particular values of $g_{0}$ and $g_{1}$ which yield either asymptotically flat or asymptotically (anti)-de Sitter solutions. The dimension of the set of solutions with an endpoint at $T_{1}$ varies greatly for different $\mathrm{g}_{0}$ and $\mathrm{g}_{1}$, but typically it is at least 3-dimensional if $\alpha_{1}>0$ and $\alpha_{2}>0$. It is possible to derive an exact class of solutions with endpoints at $T_{1}$ by looking for solutions with $V=Y$ and $W=\gamma Z$, where $\gamma$ is constant. This condition places a constraint on the remaining variables, and the requirement that the field equations preserve the constraint further fixes $\gamma$ to be the same as the ratio of $W$ to $Z$ that was found for points $T_{1,2}$. Since these exact solutions have $\bar{\lambda}=0$, however, they are of limited physical interest, and we will not list them.

* Note added: Explicit calculations show, in fact, that in the presence of a cosmological constant no asymptotically (anti)-de Sitter solutions exist with two horizons. In the case of a negative cosmological constant, asymptotically anti-de Sitter black hole solutions with a single horizon are found numerically $[25]$. 
One further technical point should be made here. It was noted in section 3 that the 5dimensional autonomous system was not valid for $\mathrm{g}_{0}=(D-3) \mathrm{g}_{1}$, since the transformation between the two sets of variables becomes degenerate for this combination of the parameters. It is possible to construct a 3-dimensional autonomous system for this combination, and proceed to examine the phase space. We have done this as a check and find roughly the same structure as for the more general system that we have described here, with the exception that some points such as $S_{1,2}$ are absent. Most importantly, the asymptotic forms of the solutions given in Tables 1 and 2 are not altered.

To conclude, we have shown that, with the exception of a pure cosmological constant, charged dilaton black holes with 'reasonable' asymptotic properties, namely an asymptocially flat or asymptotically (anti)-de Sitter behaviour, do not exist in the presence of a Liouville-type dilaton potential. This conclusion may seem trivial if one only considers series expansions of the type (2.7). However, it is a somewhat less trivial if one allows for the possibility for an asymptotic behaviour of the dilaton physically consistent with the weak coupling limit in string theory. Our conclusion is based on the observations that (i) the critical points $M_{1,2}$ are endpoints only for integral curves which correspond physically to $\mathcal{V} \equiv 0$; and (ii) none of the other critical points correspond to solutions with a 'reasonable' asymptotic behaviour. In the pure cosmological constant case critical points which are asymptotically (anti)-de Sitter do exist, and given the structure of the phase space it seems highly plausible that integral curvess with endpoints at these critical points do include a class of charged dilaton black hole spacetimes. However, although the method we have discussed is a useful tool for ruling out the existence of various solutions it does not provide an obvious way of rigourously proving the existence of solutions. Okai, who made investigations using power series [19], has also been unable to prove unequivocally that black hole solutions do exist. Perhaps a numerical approach is the best in such circumstances.

Acknowledgement: We would like to thank the Australian Research Council and the Rothmans Foundation for financial support. 


\section{APPENDIX A}

The points $S_{1,2}$ are endpoints for solutions which are of Robinson-Bertotti type. We justify this statement here by explicit derivation of such solutions. Let us suppose that there exist solutions for which $\phi=$ constant $\forall \xi$. From (2.14) and (2.15) it then follows that

$$
(D-2) X-Y-(D-3) V=0 .
$$

If we take the the derivative of this equation and use the field equations (2.16)-(2.20) we obtain the condition

$$
\Lambda W^{2}=\gamma^{2} Z^{2}, \quad \gamma^{2}=Q^{2} \mathrm{~g}_{0} /\left(\Lambda \mathrm{g}_{1}\right)
$$

Thus such solutions only exist for $\mathrm{g}_{0} /\left(\Lambda \mathrm{g}_{1}\right)>0$. Substituting back into the field equations we find that $X=Y=V$. The solution can be put in the form

$$
\begin{aligned}
\mathrm{d} s^{2}= & \left\{\gamma^{-2 \mathrm{~g}_{0}}\left[\frac{2\left(\mathrm{~g}_{0}+\mathrm{g}_{1}\right) Q^{2}}{(D-2)(D-3) \mathrm{g}_{1} \bar{\lambda}}\right]^{\mathrm{g}_{0}+\mathrm{g}_{1}}\right\}^{1 /\left[(D-3) \mathrm{g}_{1}-\mathrm{g}_{0}\right]} \\
& \times\left\{-Z^{2} \mathrm{~d} t^{2}+\frac{\left(\mathrm{g}_{0}+\mathrm{g}_{1}\right) \mathrm{d} Z^{2}}{(D-3) \mathrm{g}_{1} \bar{\lambda}\left[C+\mathrm{g}_{1}^{-1}\left((D-3) \mathrm{g}_{1}-\mathrm{g}_{0}\right) Q^{2} Z^{2}\right]}+g_{i j} \mathrm{~d} x^{i} \mathrm{~d} x^{j}\right\},
\end{aligned}
$$

where $C$ is an arbitrary constant, and we have used the freedom of rescaling $t$. Solutions exist only if $\left(\mathrm{g}_{0}+\mathrm{g}_{1}\right) /\left(\mathrm{g}_{1} \bar{\lambda}\right)>0$, or if $\bar{\lambda}=0$ in the case that $\mathrm{g}_{0}=-\mathrm{g}_{1}$. If $\left[(D-3) \mathrm{g}_{1}-\mathrm{g}_{0}\right] / \mathrm{g}_{1}>0$ and $\bar{\lambda}>0$ then the metric has the structure of a Robinson-Bertotti spacetime, namely the product of a 2-dimensional anti-de Sitter spacetime with a $(D-2)$-sphere. Similary, if $\left[(D-3) \mathrm{g}_{1}-\mathrm{g}_{0}\right] / \mathrm{g}_{1}<0$ then the $x^{i}=$ constant section is a 2-dimensional de Sitter space. 


\section{APPENDIX B}

Let us solve the field equations of the $W=0$ subsystem in the domain of outer communications $(\varepsilon=+1)$. The field equations reduce to

$$
\begin{aligned}
& \zeta^{\prime \prime}=(D-3)^{2} \bar{\lambda} e^{2 \zeta}, \\
& \eta^{\prime \prime}=2\left(\frac{D-3+\mathrm{g}_{0}^{2}}{D-2}\right) Q^{2} e^{2 \eta} \\
& \frac{-\zeta^{\prime 2}}{D-3}+\frac{\eta^{\prime 2}}{D-3+\mathrm{g}_{0}{ }^{2}}+\frac{\left(D-3+\mathrm{g}_{0}^{2}\right) c_{0}^{2}}{D-3}+(D-3) \bar{\lambda} e^{2 \zeta}-\frac{2 Q^{2}}{D-2} e^{2 \eta}=0,
\end{aligned}
$$

where $c_{0}$ is the integration constant defined by (3.1), and $\chi$ is completely determined in terms

of $\zeta$ and $\eta$ by a further intergration of (3.1). Equations (B.1) and (B.2) can be integrated directly with the result

$$
\begin{aligned}
& \zeta^{\prime 2}=(D-3)^{2}\left[\bar{\lambda} e^{2 \zeta}+\epsilon_{1} k_{1}^{2}\right] \\
& \eta^{\prime 2}=\left(D-3+\mathrm{g}_{0}^{2}\right)\left[\frac{2 Q^{2}}{D-2} e^{2 \eta}+\epsilon_{2} k_{2}^{2}\right],
\end{aligned}
$$

where $\epsilon_{1}=+1,0,-1, \epsilon_{2}=+1,0,-1$ and $k_{1}$ and $k_{2}$ are constants which on account of the constraint (B.3) must satisfy the condition

$$
(D-3) \epsilon_{1} k_{1}^{2}=\epsilon_{2} k_{2}{ }^{2}+\left(\frac{D-3+\mathrm{g}_{0}^{2}}{D-3}\right) c_{0}{ }^{2} .
$$

A further integration of (B.4) and (B.5) yields the result

$$
\begin{gathered}
\bar{\lambda} e^{2 \zeta}= \begin{cases}\frac{k_{1}{ }^{2}}{\sinh ^{2}\left[(D-3) k_{1}\left(\xi-\xi_{1}\right)\right]}, & \epsilon_{1}=+1, \\
\frac{1}{(D-3)^{2}\left(\xi-\xi_{1}\right)^{2}}, & \epsilon_{1}=0, \\
\frac{k_{1}{ }^{2}}{\sin ^{2}\left[(D-3) k_{1}\left(\xi-\xi_{1}\right)\right]}, & \epsilon_{1}=-1,\end{cases} \\
\frac{2 Q^{2}}{D-2} e^{2 \eta}= \begin{cases}\frac{k_{2}^{2}}{\sinh ^{2}\left[\sqrt{D-3+\mathrm{g}_{0}^{2}} k_{2}\left(\xi-\xi_{2}\right)\right]}, & \epsilon_{1}=+1, \\
\frac{1}{\left(D-3+\mathrm{g}_{0}^{2}\right)\left(\xi-\xi_{2}\right)^{2}}, & \epsilon_{1}=0, \\
\frac{k_{2}^{2}}{\sin ^{2}\left[\sqrt{D-3+\mathrm{g}_{0}^{2}} k_{2}\left(\xi-\xi_{2}\right)\right]}, & \epsilon_{1}=-1 .\end{cases}
\end{gathered}
$$

where $\xi_{1}$ and $\xi_{2}$ are arbitrary constants. 
To compare with the results of Gibbons and Maeda [1] let us introduce a new radial coordinate, $\bar{r}=\int e^{2 \zeta} \mathrm{d} \xi$, so that

$$
e^{2 \zeta}=(D-3)^{2} \bar{\lambda}\left(\bar{r}^{2}-\epsilon_{1} \bar{r}_{0}^{2}\right)
$$

where $(D-3) \bar{\lambda} \bar{r}_{0}=k_{1}$. In the case $\epsilon_{1}=+1, \epsilon_{2}=+1$, the metric functions are found to be

$$
\begin{aligned}
& e^{2 u} \propto\left(\frac{\bar{r}-\bar{r}_{0}}{\bar{r}+\bar{r}_{0}}\right)^{\frac{c_{0} \mathrm{~g}_{0}}{(D-3) k_{1}}}\left\{\left(\frac{\bar{r}-\bar{r}_{0}}{\bar{r}+\bar{r}_{0}}\right)^{c}-A^{2}\left(\frac{\bar{r}-\bar{r}_{0}}{\bar{r}+\bar{r}_{0}}\right)^{-c}\right\}^{\frac{-2(D-3)}{D-3+g_{0}{ }^{2}}}, \\
& R^{D-3} \propto\left(\bar{r}+\bar{r}_{0}\right)\left(\frac{\bar{r}-\bar{r}_{0}}{\bar{r}+\bar{r}_{0}}\right)^{\frac{(D-3) k_{1}-c_{0} \mathrm{~g}_{0}}{2(D-3) k_{1}}}\left\{\left(\frac{\bar{r}-\bar{r}_{0}}{\bar{r}+\bar{r}_{0}}\right)^{c}-A^{2}\left(\frac{\bar{r}-\bar{r}_{0}}{\bar{r}+\bar{r}_{0}}\right)^{-c}\right\}^{\frac{D-3}{D-3+\mathrm{g}_{0}{ }^{2}}}, \\
& \mathrm{e}^{2 \phi}=\left(\frac{\bar{r}-\bar{r}_{0}}{\bar{r}+\bar{r}_{0}}\right)^{\frac{-(D-2) c_{0}}{2(D-3) k_{1}}}\left\{\left(\frac{\bar{r}-\bar{r}_{0}}{\bar{r}+\bar{r}_{0}}\right)^{c}-A^{2}\left(\frac{\bar{r}-\bar{r}_{0}}{\bar{r}+\bar{r}_{0}}\right)^{-c}\right\}^{\frac{-(D-2) g_{0}}{D-3+\mathrm{g}_{0}{ }^{2}}},
\end{aligned}
$$

where the constants $c$ and $A$ are given by

$$
\begin{aligned}
c & =\frac{k_{2} \sqrt{D-3+\mathrm{g}_{0}^{2}}}{2 k_{1}(D-3)}, \\
A & =\exp \left[\sqrt{D-3+\mathrm{g}_{0}^{2}} k_{2}\left(\xi_{2}-\xi_{1}\right)\right] .
\end{aligned}
$$

One may verify that setting $k_{2}=(D-3) k_{1} /\left(D-3+\mathrm{g}_{0}{ }^{2}\right)^{1 / 2}$, and hence $c=\frac{1}{2}$ and by (B.6) $c_{0}=(D-3) \mathrm{g}_{0} k_{1} /\left(D-3+\mathrm{g}_{0}^{2}\right)$, one obtains the familiar Gibbons-Maeda charged dilaton black hole [1].

Now consider the case $\epsilon_{1}=0, \epsilon_{2}=0$. One then finds that

$$
\begin{aligned}
& e^{2 u} \propto\left(\frac{\bar{r}}{\bar{a} \bar{r}+1}\right)^{\frac{2(D-3)}{D-3+\mathrm{g}_{0}{ }^{2}}}, \\
& R^{D-3} \propto \bar{r}\left(\frac{\bar{r}}{\bar{a} \bar{r}+1}\right)^{\frac{-(D-3)}{D-3+\mathrm{g}_{0}{ }^{2}}},
\end{aligned}
$$

\footnotetext{
* This coordinate is denoted ' $\eta$ ' in the notation of [1].
} 


$$
e^{2 \phi} \propto\left(\frac{\bar{r}}{\bar{a} \bar{r}+1}\right)^{\frac{(D-2) \mathrm{g}_{0}}{D-3+\mathrm{g}_{0}{ }^{2}}}
$$

where $\bar{a}=\bar{\lambda}(D-3)^{2}\left(\xi_{2}-\xi_{1}\right)^{2}$. If $\bar{a} \neq 0$ then these solutions approach the points $M_{1,2}$ as $\bar{r} \rightarrow \infty$, while if $\bar{a}=0$ they approach $K_{1,2}$ as $\bar{r} \rightarrow \infty$. It is clear that these solutions represent naked singularites, and hence have limited physical interest. It is straightforward to show that the solutions obtained in the case $\epsilon_{1}=-1, \epsilon_{2}=-1$ similarly approach either $M_{1,2}$ or $K_{1,2}$ as $\bar{r} \rightarrow \infty$.

\section{REFERENCES}

1. G.W. Gibbons and K. Maeda, Nucl. Phys. B298 (1988) 741.

2. D. Garfinkle, G.T. Horowitz and A. Strominger, Phys. Rev. D43 (1991) 3140; err. ibid. 45 (1992) 3888.

3. B. Harms and Y. Leblanc, Phys. Rev. D46 (1992) 2334.

4. C.F.E. Holzhey and F. Wilczek, Nucl. Phys. B380 (1992) 447.

5. T. Damour and A.M. Polyakov, Nucl. Phys. B423 (1994) 532.

6. R. Gregory and J.A. Harvey 1993, Phys. Rev. D47 (1993) 2411.

7. J.H. Horne and G.T. Horowitz 1993, Nucl. Phys. B399 (1993) 169.

8. J.P. Derendinger, L.E. Ibáñez and H.P. Nilles, Phys. Lett. 155B (1985) 65;

M. Dine, R. Rohm, N. Seiberg and E. Witten, Phys. Lett. 156B (1985) 55.

9. S. Kalara and K.A. Olive, Phys. Lett. 218B (1989) 148.

M.C. Bento, O. Bertolami and P.M. Sá, Phys. Lett. 262B (1991) 11; Mod. Phys. Lett. A7 (1992), 911.

M. Gasperini and G. Veneziano, Phys. Lett. 277B (1992) 256.

A.A. Tseytlin and C. Vafa, Nucl. Phys. B372 (1992) 443.

A.A. Tseytlin, Class. Quantum Grav. 9 (1992) 979; Int. J. Mod. Phys. D1 (1992) 223.

J. García-Bellido and M. Quirós, Nucl. Phys. B368 (1992) 463; Nucl. Phys. B385 (1992) 558.

N. Kaloper and K.A. Olive, Astropart. Phys. 1 (1993) 185.

10. S. Mignemi and D.L. Wiltshire, Class. Quantum Grav. 6 (1989) 987.

11. D.L. Wiltshire, Phys. Rev. D44 (1991) 1100.

12. S. Mignemi and D.L. Wiltshire, Phys. Rev. D46 (1992) 1475. 
13. F. Belgiorno and A.S. Cattaneo, "Black holes and cosmological constant in bosonic string theory: some remarks", Preprint IFUM 450/FT, hep-th/9309156.

14. S.K. Rama, "Cosmological constant in low energy $d=4$ string leads to naked singularity", Preprint TCD-1-94, hep-th/9402009.

15. T. Maki and K. Shiraishi, Class. Quantum Grav. 10 (1993) 2171.

16. D. Kastor and J. Traschen, Phys. Rev. D47 (1993) 5370.

17. M. Cadoni and S. Mignemi, Phys. Rev. D48 (1993) 5536; Nucl. Phys. B427 (1994) 669.

18. M. Cvetič and A.A. Tseytlin, Nucl. Phys. B416 (1994) 137.

19. T. Okai, "4-dimensional dilaton black holes with cosmological constant", Preprint UT-679, hep-th/9406126

20. J.E. Chase, Commun. Math. Phys. 19 (1970) 276.

21. J.D. Bekenstein, Phys. Rev. D5 (1972) 1239; 2403.

22. G.W. Gibbons "Self-gravitating magnetic monopoles, global monopoles and black holes", in J.D. Barrow, A.B. Henriques, M.T.V.T. Lago and M.S. Longair (eds.), The Physical Universe: Proceedings of the XII Autumn School, Lisbon, 1990, (Springer, Berlin, 1991).

23. G.W. Gibbons and D.L. Wiltshire, Ann. Phys. (N.Y.) 167 (1986) 201; err. ibid. 176 (1987) 393.

24. H. Buchdahl, Phys. Rev. 115 (1959) 1325.

25. S.J. Poletti, J. Twamley and D.L. Wiltshire, preprint ADP-94-17/M24, hep-th/9412076.

\section{FIGURE CAPTIONS}

Fig. 1. The hemisphere at infinity in the reduced $W=0(\Lambda=0)$ subspace in terms of the coordinates $\theta_{1}, \phi_{1}$ defined by (3.7). Although these integral curves do not correspond to physical solutions it is nevertheless helpful to sketch them since by continuity arguments they will determine the behaviour of the physical integral curves which lie within the sphere at infinity but near its surface. 
This figure "fig1-1.png" is available in "png" format from: http://arxiv.org/ps/gr-qc/9407021v2 